Stud. Univ. Babeş-Bolyai Math. 62(2017), No. 1, 127-135

DOI: $10.24193 /$ subbmath.2017.0010

\title{
Ball convergence of a stable fourth-order family for solving nonlinear systems under weak conditions
}

\author{
Ioannis K. Argyros, Munish Kansal and Vinay Kanwar
}

\begin{abstract}
We present a local convergence analysis of fourth-order methods in order to approximate a locally unique solution of a nonlinear equation in Banach space setting. Earlier studies have shown convergence using Taylor expansions and hypotheses reaching up to the fifth derivative although only the first derivative appears in these methods. We only show convergence using hypotheses on the first derivative. We also provide computable: error bounds, radii of convergence as well as uniqueness of the solution with results based on Lipschitz constants not given in earlier studies. The computational order of convergence is also used to determine the order of convergence. Finally, numerical examples are also provided to show that our results apply to solve equations in cases where earlier studies cannot apply.
\end{abstract}

Mathematics Subject Classification (2010): 65D10, 65D99.

Keywords: Local convergence, nonlinear equation, Lipschitz condition, Fréchet derivative.

\section{Introduction}

Let $B_{1}, B_{2}$ be Banach spaces and $D$ be a convex subset of $B_{1}$. Let also $L\left(B_{1}, B_{2}\right)$ denote the space of bounded linear operators from $B_{1}$ into $B_{2}$.

In the present paper, we deal with the problem of approximating a locally unique solution $x^{*}$ of the equation

$$
F(x)=0,
$$

where $F: D \subseteq B_{1} \rightarrow B_{2}$ is a Fréchet-differentiable operator.

Numerous problems can be written in the form of (1.1) using Mathematical Modelling [3, 5, 8, 9, 12, 13, 18, 19, 22, 26, 28, 29, 30]. Analytical methods for solving such problems are almost non-existent and therefore, it is only possible to obtain approximate solutions by relying on numerical methods based on iterative procedure 
[1-24]. In particular, we present the local convergence of the methods studied in [14] and defined for each $n=0,1,2,3, \ldots$ by

$$
\left\{\begin{array}{l}
y_{n}=x_{n}-F^{\prime}\left(x_{n}\right)^{-1} F\left(x_{n}\right), \\
z_{n}=y_{n}-\frac{1}{\beta} F^{\prime}\left(x_{n}\right)^{-1} F\left(y_{n}\right), \\
x_{n+1}=z_{n}-F^{\prime}\left(x_{n}\right)^{-1}\left(\alpha F\left(y_{n}\right)+\beta F\left(z_{n}\right)\right),
\end{array}\right.
$$

where $\alpha=2-\frac{1}{\beta}-\beta, \beta \in \mathbb{R} \backslash\{0\}$ and $\alpha \in \mathbb{R}$.

Method (1.2) has fourth-order of convergence, except for $\beta=1 / 5$. For this particular value, method attains fifth-order of convergence. The fourth order of convergence was based on Taylor expansions and hypotheses reaching up to the fifth derivative of function $F$ although only the first derivative appears in these methods. Moreover, no computable error bounds on the distances $\left\|x_{n}-x^{*}\right\|$ or uniqueness results or compuatble radius of convergence were given. These problems reduce the applicability of these methods.

As a motivational example, define function $F$ on $D=\left[\frac{-1}{2}, \frac{5}{2}\right]$ by

$$
F(x)=\left\{\begin{array}{lr}
x^{3} \ln x^{2}+x^{5}-x^{4}, & x \neq 0, \\
0, & x=0 .
\end{array}\right.
$$

Choose $x^{*}=1$. We have that

$$
\begin{aligned}
& F^{\prime}(x)=3 x^{2} \ln x^{2}+5 x^{4}-4 x^{3}+2 x^{2}, \\
& F^{\prime \prime}(x)=6 x \ln x^{2}+20 x^{3}-12 x^{2}+10 x, \\
& F^{\prime \prime \prime}(x)=6 \ln x^{2}+60 x^{2}-24 x+22 .
\end{aligned}
$$

Then, the results in [14] cannot be used to solve the equation $F(x)=0$, since function $F^{\prime \prime \prime}$ is unbounded on $D$.

In the present study, we only use hypotheses on the first derivative and find error bounds, radii of convergence and uniqueness results based on Lipschitz constants. Moreover, since we avoid derivatives of order higher than one, we compute the computational order of convergence which does not require the knowledge of $x^{*}$ or the existence of high order derivatives. This way we expand the applicability of these methods.

The rest of the paper is organized as follows: The local convergence of both methods is given in Section 2, whereas numerical examples are provided in the concluding Section 3.

\section{Local convergence}

We present the local convergence analysis of method (1.2) in this section.

The local convergence analysis is based on some scalar functions and parameters. Let $L_{0}>0, L>0, M \geq 1, \beta \in \mathbb{R} \backslash\{0\}$ and $\alpha \in \mathbb{R}$ be given parameters. Define function 
$g_{1}, g_{2}, h_{2}, g_{3}$ and $h_{3}$ on the interval $\left[0, \frac{1}{L_{0}}\right)$ by

$$
\begin{aligned}
& g_{1}(t)=\frac{L t}{2\left(1-L_{0} t\right)}, \\
& g_{2}(t)=\left(1+\frac{M}{|\beta|\left(1-L_{0} t\right)}\right) g_{1}(t), \\
& h_{2}(t)=g_{2}(t)-1, \\
& g_{3}(t)=g_{2}(t)+\frac{M}{1-L_{0} t}\left(|\alpha| g_{1}(t)+|\beta| g_{2}(t)\right), \\
& h_{3}(t)=g_{3}(t)-1
\end{aligned}
$$

and parameter $r_{A}$ by

$$
r_{A}=\frac{2}{2 L_{0}+L}
$$

We have that $g_{1}\left(r_{A}\right)=1$ and $0 \leq g_{1}(t)<1$ for each $t \in\left[0, r_{A}\right)$.

We also get that $h_{2}(0)=h_{3}(t)=-1<0$ and $h_{2}(t) \rightarrow+\infty, h_{3}(t) \rightarrow+\infty$ as $t \rightarrow \frac{1^{-}}{L_{0}}$. It follows from intermediate value theorem that functions $h_{2}$ and $h_{3}$ have zeros in the interval $\left(0, \frac{1}{L_{0}}\right)$. Denote by $r_{2}$ and $r_{3}$ the smallest such zeros.

Define the convergence radius $r$ by

$$
r=\min \left\{r_{A}, r_{2}, r_{3}\right\}
$$

Then, we have that

$$
0<r \leq r_{A}
$$

and

$$
0 \leq g_{i}(t)<1, i=1,2,3 .
$$

Let $U(v, \rho)$ and $\bar{U}(v, \rho)$ stand, respectively for the open and closed balls in $B_{1}$ with center $v \in B_{1}$ and of radius $\rho>0$. Next, we present the local convergence analysis of method (1.2) using the preceding notation.

Theorem 2.1. Let $F: D \subseteq B_{1} \rightarrow B_{2}$ be a Fréchet-differentiable operator. Suppose that there exist $x^{*} \in D$ and $L_{0}>0$ such that for each $x \in D$

$$
F\left(x^{*}\right)=0, F^{\prime}\left(x^{*}\right)^{-1} \in L\left(B_{2}, B_{1}\right),
$$

and

$$
\left\|F^{\prime}\left(x^{*}\right)^{-1}\left(F^{\prime}(x)-F^{\prime}\left(x^{*}\right)\right)\right\| \leq L_{0}\left\|x-x^{*}\right\| .
$$

Moreover, suppose that there exist constants $L>0$ and $M \geq 1$ such that for each $x, y \in D_{0}:=D \cap U\left(x^{*}, \frac{1}{L_{0}}\right)$

$$
\begin{gathered}
\left\|F^{\prime}\left(x^{*}\right)^{-1}\left(F^{\prime}(x)-F^{\prime}(y)\right)\right\| \leq L\|x-y\|, \\
\left\|F^{\prime}\left(x^{*}\right)^{-1} F^{\prime}(x)\right\| \leq M
\end{gathered}
$$

and

$$
\bar{U}\left(x^{*}, r\right) \subseteq D
$$

where the radius of convergence $r$ is defined by (2.1). Then, the sequence $\left\{x_{n}\right\}$ generated for $x_{0} \in U\left(x^{*}, r\right)-\left\{x^{*}\right\}$ by method (1.2) is well defined, remains in $U\left(x^{*}, r\right)$ and 
converges to the solution $x^{*}$ of equation $F(x)=0$. Moreover, the following estimates hold

$$
\begin{gathered}
\left\|y_{n}-x^{*}\right\| \leq g_{1}\left(\left\|x_{n}-x^{*}\right\|\right)\left\|x_{n}-x^{*}\right\| \leq\left\|x_{n}-x^{*}\right\|<r, \\
\left\|z_{n}-x^{*}\right\| \leq g_{2}\left(\left\|x_{n}-x^{*}\right\|\right)\left\|x_{n}-x^{*}\right\| \leq\left\|x_{n}-x^{*}\right\|
\end{gathered}
$$

and

$$
\left\|x_{n+1}-x^{*}\right\| \leq g_{3}\left(\left\|x_{n}-x^{*}\right\|\right)\left\|x_{n}-x^{*}\right\| \leq\left\|x_{n}-x^{*}\right\|,
$$

where the " $g$ " functions are defined previously. Furthermore, for $T \in\left[r, \frac{2}{L_{0}}\right)$, the limit point $x^{*}$ is the only solution of $F(x)=0$ in $D_{1}:=U\left(x^{*}, T\right) \cap D$.

Proof. We shall show estimates (2.9)-(2.11) using mathematical induction. By hypothesis $x_{0} \in U\left(x^{*}, r\right)-\left\{x^{*}\right\},(2.1),(2.4)$ and (2.5), we have that

$$
\left\|F^{\prime}\left(x^{*}\right)^{-1}\left(F^{\prime}\left(x_{0}\right)-F^{\prime}\left(x^{*}\right)\right)\right\| \leq L_{0}\left\|x_{0}-x^{*}\right\|<L_{0} r<1 .
$$

It follows from (2.12) and the Banach lemma on invertible functions [7, 26, 28, 30] that $F^{\prime}\left(x_{0}\right)^{-1} \in L\left(B_{2}, B_{1}\right)$ and

$$
\left\|F^{\prime}\left(x_{0}\right)^{-1} F^{\prime}\left(x^{*}\right)\right\| \leq \frac{1}{1-L_{0}\left\|x_{0}-x^{*}\right\|} .
$$

Hence, $y_{0}, z_{0}, x_{1}$ are well defined by method (1.2) for $n=0$. We can have that

$$
y_{0}-x^{*}=x_{0}-x^{*}-F^{\prime}\left(x_{0}\right)^{-1} F\left(x_{0}\right) \text {. }
$$

Using (2.1), (2.2), (2.3) (for $i=1),(2.6),(2.13)$ and (2.14), we obtain in turn that

$$
\begin{aligned}
\left\|y_{0}-x^{*}\right\|= & \left\|x_{0}-x^{*}-F^{\prime}\left(x_{0}\right)^{-1} F\left(x_{0}\right)\right\| \leq\left\|F^{\prime}\left(x_{0}\right)^{-1} F^{\prime}\left(x^{*}\right)\right\| \\
& \left\|\int_{0}^{1} F^{\prime}\left(x^{*}\right)^{-1}\left(F^{\prime}\left(x^{*}+\theta\left(x_{0}-x^{*}\right)\right)-F^{\prime}\left(x_{0}\right)\right)\left(x_{0}-x^{*}\right) d \theta\right\| \\
\leq & \frac{L\left\|x_{0}-x^{*}\right\|^{2}}{2\left(1-L_{0}\left\|x_{0}-x^{*}\right\|\right)} \\
= & g_{1}\left(\left\|x_{0}-x^{*}\right\|\right)\left\|x_{0}-x^{*}\right\| \leq\left\|x_{0}-x^{*}\right\|<r,
\end{aligned}
$$

which shows $(2.9)$ for $n=0$ and $y_{0} \in U\left(x^{*}, r\right)$. We also have that

$$
F\left(x_{0}\right)=F\left(x_{0}\right)-F\left(x^{*}\right)=\int_{0}^{1} F^{\prime}\left(x^{*}+\theta\left(x_{0}-x^{*}\right)\right)\left(x_{0}-x^{*}\right) d \theta .
$$

Notice that $\left\|x^{*}+\theta\left(x_{0}-x^{*}\right)-x^{*}\right\|=\theta\left\|x_{0}-x^{*}\right\|<r$, so $x^{*}+\theta\left(x_{0}-x^{*}\right) \in U\left(x^{*}, r\right)$. Then, by (2.7) and (2.16), we get that

$$
\left\|F^{\prime}\left(x^{*}\right)^{-1} F\left(x_{0}\right)\right\| \leq M\left\|x_{0}-x^{*}\right\| .
$$

In view of $(2.1),(2.2),(2.3)$ (for $i=2),(2.13),(2.15)$ and $(2.17)$ (for $\left.x_{0}=y_{0}\right)$, we get that

$$
\begin{aligned}
\left\|z_{0}-x^{*}\right\| & \leq\left\|y_{0}-x^{*}\right\|+\frac{M\left\|y_{0}-x^{*}\right\|}{|\beta|\left(1-L_{0}\left\|x_{0}-x^{*}\right\|\right)} \\
& \left.\leq\left(1+\frac{M}{|\beta|\left(1-L_{0}\left\|x_{0}-x^{*}\right\|\right)}\right) g_{1}\left(\left\|x_{0}-x^{*}\right\|\right)\right)\left\|x_{0}-x^{*}\right\| \\
& \left.=g_{2}\left(\left\|x_{0}-x^{*}\right\|\right)\right)\left\|x_{0}-x^{*}\right\| \leq\left\|x_{0}-x^{*}\right\|<r,
\end{aligned}
$$


which shows (2.10) for $n=0$ and $z_{0} \in U\left(x^{*}, r\right)$. By (2.1), (2.2), (2.3) (for $i=3$ ), (2.13), (2.15) and (2.17) (for $\left.x_{0}=y_{0}\right)$, we get that

$$
\begin{aligned}
\left\|x_{1}-x^{*}\right\| & \leq\left\|z_{0}-x^{*}\right\|+\frac{M}{1-L_{0}\left(\left\|x_{0}-x^{*}\right\|\right)}\left(|\alpha|\left\|y_{0}-x^{*}\right\|+|\beta|\left\|z_{0}-x^{*}\right\|\right) \\
& \leq\left[g_{2}\left(\left\|x_{0}-x^{*}\right\|\right)+\frac{M}{1-L_{0}\left(\left\|x_{0}-x^{*}\right\|\right)}\left(|\alpha| g_{1}\left(\left\|x_{0}-x^{*}\right\|\right)\right.\right. \\
& \left.\left.+|\beta| g_{2}\left(\left\|x_{0}-x^{*}\right\|\right)\right)\right]\left\|x_{0}-x^{*}\right\| \\
& =g_{3}\left(\left\|x_{0}-x^{*}\right\|\right)\left\|x_{0}-x^{*}\right\|<\left\|x_{0}-x^{*}\right\|<r,
\end{aligned}
$$

which shows (2.11) for $n=0$ and $x_{1} \in U\left(x^{*}, r\right)$. By simply replacing $x_{0}, y_{0}, x_{1}$ by $x_{n}, y_{n}, x_{n+1}$ in the preceding estimates, we complete the induction for estimates $(2.9)$ (2.11). Then, in view of the estimate

$$
\left\|x_{n+1}-x^{*}\right\| \leq c\left\|x_{n}-x^{*}\right\|<r, \quad c=g_{3}\left(\left\|x_{0}-x^{*}\right\|\right) \in[0,1),
$$

we deduce that $\lim _{n \rightarrow \infty} x_{n}=x^{*}$ and $x_{n+1} \in U\left(x^{*}, r\right)$. Finally, to show the uniqueness part, let $y^{*} \in D_{1}$ with $F\left(y^{*}\right)=0$. Define

$$
Q=\int_{0}^{1} F^{\prime}\left(x^{*}+\theta\left(y^{*}-x^{*}\right) d \theta .\right.
$$

Using (2.5), we get that

$$
\left\|F^{\prime}\left(x^{*}\right)^{-1}\left(Q-F^{\prime}\left(x^{*}\right)\right)\right\| \leq \frac{L_{0}}{2}\left\|x^{*}-y^{*}\right\| \leq \frac{L_{0}}{2} T<1 .
$$

Hence, $Q^{-1} \in L\left(B_{2}, B_{1}\right)$. Then, by the identity $0=F\left(y^{*}\right)-F\left(x^{*}\right)=Q\left(y^{*}-x^{*}\right)$, we conclude that $x^{*}=y^{*}$.

Remark 2.2. $\quad$ 1. The condition (2.7) can be dropped, since this condition follows from (2.5), if we set

$$
M(t)=1+L_{0} t
$$

or

$$
M(t)=M=2,
$$

since $t \in\left[0, \frac{1}{L_{0}}\right)$.

2. The results obtained here can also be used for operators $F$ satisfying autonomous differential equations $[5,7]$ of the form:

$$
F^{\prime}(x)=P(F(x)),
$$

where $P$ is a continuous operator. Then, since $F^{\prime}\left(x^{*}\right)=P\left(F\left(x^{*}\right)\right)=P(0)$, we can apply the results without actually knowing $x^{*}$. For example, let $F(x)=e^{x}-1$. Then, we can choose $P(x)=x+1$.

3. The radius $r_{A}^{-}=\frac{2}{2 L_{0}+L_{1}}$ was shown by us to be the convergence radius of Newton's method [5]

$$
x_{n+1}=x_{n}-F^{\prime}\left(x_{n}\right)^{-1} F\left(x_{n}\right), \text { for each } n=0,1,2, \ldots
$$


provided the conditions $(2.4)-(2.6)$ hold on $D$. Let $L_{1}$ be the corresponding to $L$ constant. It follows from the definition of $r$ that the convergence radius $r$ of the method (1.2) cannot be larger than the convergence radius $r_{A}^{-}$of the second order Newton's method (3.3). As already noted in [5], $r_{A}^{-}$is at least as large as the convergence ball given by Rheinboldt [28]

$$
r_{R}=\frac{2}{3 L_{1}} .
$$

In particular, for $L_{0}<L_{1}$, we have that

$$
r_{R}<r_{1}
$$

and

$$
\frac{r_{R}}{r_{A}^{-}} \rightarrow \frac{1}{3} \text { as } \frac{L_{0}}{L_{1}} \rightarrow 0 \text {. }
$$

That is our convergence ball $r_{A}^{-}$is atmost three times larger than Rheinboldt's. The same value of $r_{R}$ was given by Traub [30]. Notice that $L \leq L_{1}$, since $D_{0} \subseteq D$. Therefore, $r_{A}^{-} \leq r_{A}$.

4. It is worth noticing that method (1.2) is not changing when we use the conditions of Theorem 2.1 instead of stronger conditions used in [14]. Moreover, we can compute the computational order of convergence (COC) defined by

$$
\xi^{*}=\sup \frac{\ln \left(\frac{\left\|x_{n+1}-x^{*}\right\|}{\left\|x_{n}-x^{*}\right\|}\right)}{\ln \left(\frac{\left\|x_{n}-x^{*}\right\|}{\left\|x_{n-1}-x^{*}\right\|}\right)},
$$

or the approximate computational order of convergence (ACOC) defined by

$$
\xi=\sup \frac{\ln \left(\frac{\left\|x_{n+1}-x_{n}\right\|}{\left\|x_{n}-x_{n-1}\right\|}\right)}{\ln \left(\frac{\left\|x_{n}-x_{n-1}\right\|}{\left\|x_{n-1}-x_{n-2}\right\|}\right)} .
$$

This way we obtain in practice the order of convergence in a way that avoids the bounds involving estimates using estimates higher than the first Fréchet derivative of operator $F$. Notice also that the computation of $\xi$ does not require knowledge of $x^{*}$.

\section{Numerical examples}

We present numerical examples in this section.

Example 3.1. Let $X=Y=\mathbb{R}^{3}, D=\bar{U}(0,1), x^{*}=(0,0,0)^{T}$. Define function $F$ on $D$ for $w=(x, y, z)^{T}$ by

$$
F(w)=\left(e^{x}-1, \frac{e-1}{2} y^{2}+y, z\right)^{T} .
$$

Then, the Fréchet derivative is given by

$$
F^{\prime}(w)=\left[\begin{array}{ccc}
e^{x} & 0 & 0 \\
0 & (e-1) y+1 & 0 \\
0 & 0 & 1
\end{array}\right]
$$


We have that $L_{0}=e-1, L=e^{\frac{1}{L_{0}}}=1.789572397, M=e^{\frac{1}{L_{0}}}=1.7896$ and $L_{1}=e$. The parameters using method (1.2) are:

$$
r_{A}=0.382692, r_{2}=0.145318, r_{3}=0.0826175, r=0.0826175, r_{A}^{-}=0.324947 .
$$

Example 3.2. Let $B_{1}=B_{2}=C[0,1]$, the space of continuous functions defined on $[0,1]$ and be equipped with the max norm. Let $D=\bar{U}(0,1)$ and $B(x)=F^{\prime \prime}(x)$ for each $x \in D$. Define function $F$ on $D$ by

$$
F(\phi)(x)=\phi(x)-5 \int_{0}^{1} x \theta \phi(\theta)^{3} d \theta .
$$

We have that

$$
F^{\prime}(\phi(\xi))(x)=\xi(x)-15 \int_{0}^{1} x \theta \phi(\theta)^{2} \xi(\theta) d \theta, \text { for each } \xi \in D .
$$

Then, we get that $x^{*}=0, L_{0}=7.5, L_{1}=15, L=15, M=2$. The parameters using method (1.2) are:

$$
r_{A}=0.0666667, r_{2}=0.0198959, r_{3}=0.0101189, r=0.0101189, r_{A}^{-}=0.0666667 \text {. }
$$

Example 3.3. Let $B_{1}=B_{2}=\mathbb{R}, D=\bar{U}(0,1)$. Define $F$ on $D$ by

$$
F(x)=e^{x}-1 \text {. }
$$

Then, $F^{\prime}(x)=e^{x}$ and $\xi=0$. We get that $L_{0}=e-1<L=e^{\frac{1}{L_{0}}}<L_{1}=e$ and $M=2$. Then, for method (1.2) the parameters are:

$$
\begin{gathered}
r_{A}=0.382692, r_{2}=0.13708, r_{3}=0.0742433, \\
r=0.0742433, r_{A}^{-}=0.324947, \xi=3.8732 .
\end{gathered}
$$

Example 3.4. Let $B_{1}=B_{2}=\mathbb{R}$ and define function $F$ on $D=\mathbb{R}$ by

$$
F(x)=\beta x-\gamma \sin (x)-\delta,
$$

where $\beta, \gamma, \delta$ are given real numbers. Suppose that there exists a solution $\xi$ of $F(x)=0$ with $F^{\prime}(\xi) \neq 0$. Then, we have

$$
L_{1}=L_{0}=L=\frac{|\gamma|}{|\beta-\gamma \cos \xi|}, M=\frac{|\gamma|+|\beta|}{|\beta-\gamma \cos \xi|} .
$$

Then one can find the convergence radii for different values of $\beta, \gamma$ and $\delta$. As a specific example, let us consider Kepler's equation (3.3) with $\beta=1,0 \leq \gamma<1$ and $0 \leq \delta \leq \pi$. A numerical study was presented in [15] for different values of $\gamma$ and $\delta$. Let us take $\gamma=0.9$ and $\delta=0.1$. Then the solution is given by $x^{*}=0.6308435$.

Hence, for method (1.2) the parameters are:

$$
\begin{gathered}
r_{A}=0.202387, r_{2}=0.032669, r_{3}=0.00804637, \\
r=0.00804637, r_{A}^{-}=0.202387, \xi=4.0398 .
\end{gathered}
$$


Example 3.5. Returning back to the motivational example at the introduction of this paper, we have that $L=L_{0}=146.6629073, M=2, L_{1}=L$. The parameters using method (1.2) are:

$$
\begin{gathered}
r_{A}=0.00689682, r_{2}=0.0033639187, r_{3}=0.00230533728667086, \\
r=0.00230533728667086, r_{A}^{-}=0.00689682 \text { and } \xi=3.4324 .
\end{gathered}
$$

\section{References}

[1] Adomian, G., Solving Frontier problem of physics: The decomposition method, Kluwer Academic Publishers, Dordrechet, 1994.

[2] Amat, S., Busquier, S., Guttiérrez, J.M., Geometric constructions of iterative functions to solve nonlinear equations, J. Comput. Appl. Math., 157(2003), 197-205.

[3] Amat, S., Busquier, S., Plaza, S., Dynamics of the King's and Jarratt iterations, Aequationes. Math., 69(2005), 212-213.

[4] Amat, S., Hernández, M.A., Romero, N., A modified Chebyshev's iterative method with at least sixth order of convergence, Appl. Math. Comput., 206(1)(2008), 164-174.

[5] Argyros, I.K., Convergence and Applications of Newton-type Iterations, Springer, 2008.

[6] Argyros, I.K., Chen D., Quian, Q., The Jarratt method in Banach space setting, J. Comput. Appl. Math., 51(1994), 103-106.

[7] Argyros, I.K., Hilout S., Computational Methods in Nonlinear Analysis, World Scientific Publ. Comp. New Jersey, 2013.

[8] Argyros, I.K., Magreñán, A.A., Ball convergence theorems and the convergence planes of an iterative method for nonlinear equations, SeMA, 71(1)(2015), 39-55.

[9] Argyros, I.K., George, S., Local convergence of some high-order Newton-like method with frozen derivatives, SeMA. doi: 10.1007/s40324-015-00398-8.

[10] Babolian, E., Biazar, J., Vahidi, A.R., Solution of a system of nonlinear equations by Adomian decomposition method, Appl. Math. Comput., 150(2004), 847-854.

[11] Cordero, A., Torregrosa, J.R., Variants of Newton's method using fifth-order quadrature formulas, Appl. Math. Comput., 190(2007), 686-698.

[12] Cordero, A., Torregrosa, J.R., Variants of Newton's method for functions of several variables, Appl. Math. Comput., 183(2006), 199-208.

[13] Cordero, A., Torregrosa, J.R., Vassileva, M.P., Increasing the order of convergence of iterative schemes for solving nonlinear systems, J. Comput. Appl. Math., 252(2012), 86-94.

[14] Cordero, A., Gutiérrez, J.M., Magreñán, A.A., Torregrosa, J.R., Stability analysis of a parametric family of iterative methods for solving nonlinear models, Appl. Math. Comput. (to appear).

[15] Danby, J.M.A., Burkardt, T.M., The solution of Kepler's equation, I. Celest. Mech., 31(1983), 95-107.

[16] Darvishi, M.T., Barati, A., A third-order Newton-type method to solve systems of nonlinear equations, Appl. Math. Comput., 187(2007), 630-635.

[17] Darvishi, M.T., Barati, A., Super cubic iterative methods to solve systems of nonlinear equations, Appl. Math. Comput., 188(2007), 1678-1685. 
[18] Ezquerro, J.A., Hernández, M.A., New iterations of $R$-order four with reduced computational cost, BIT Numer Math., 49(2009), 325-342.

[19] Ezquerro, J.A., Hernández, M.A., A uniparametric halley type iteration with free second derivative, Int. J. Pure and Appl. Math., 6(1)(2003), 99-110.

[20] Golbabai, A., Javidi, M., A new family of iterative methods for solving system of nonlinear algebraic equations, Appl. Math. Comput., 190(2007), 1717-1722.

[21] Gutiérrez, J.M., Hernández, M.A., Recurrence relations for the super-Halley method, Comput. Math. Appl., 36(1998), 1-8.

[22] Kou, J., A third-order modification of Newton method for systems of nonlinear equations, Appl. Math. Comput., 191(2007), 117-121.

[23] Montazeri, H., Soleymani, F., Shateyi, S., Motsa, S.S., On a new method for computing the numerical solution of systems of nonlinear equations, J. Appl. Math. 2012, Article ID 751975 .

[24] Noor, M.A., Waseem M., Some iterative methods for solving a system of nonlinear equations, Comput. Math. Appl., 57(2009), 101-106.

[25] Petković, M.S., Neta, B., Petković, L.D., Dzunić, J., Multipoint Methods for Solving Nonlinear Equations, Elsevier, Amsterdam, 2013.

[26] Potra, F.A., Pták, V., Nondiscrete introduction and iterative processes, Research Notes in Mathematics, Pitman, Boston, MA, 103(1984).

[27] Qifang, Su., A unified model for solving a system of nonlinear equations, Appl. Math. Comput. (to appear).

[28] Rheinboldt, W.C., An adaptive continuation process for solving systems of nonlinear equations, Mathematical Models and Numerical Methods (A.N. Tikhonov et al. eds.), Banach Center, Warsaw, Poland, 3(19), 129-142.

[29] Sharma, J.R., Guha, R.K., Sharma, R., An efficient fourth-order weighted-Newton method for systems of nonlinear equations, Numer. Algor., 62(2013), 307-323.

[30] Traub, J.F., Iterative Methods for the Solution of Equations, Prentice-Hall, New Jersey, 1964.

Ioannis K. Argyros

Department of Mathematical Sciences

Cameron University, Lawton, OK 73505, USA

e-mail: iargyros@cameron.edu

Munish Kansal

(Corresponding author)

University Institute of Engineering and Technology

Panjab University, Chandigarh-160-014, India

e-mail: mkmaths@gmail.com

Vinay Kanwar

University Institute of Engineering and Technology

Panjab University, Chandigarh-160-014, India 